\title{
The Association Between Mentor-Mentee Network Features and Publication Productivity Among Early Career Academic Generalists
}

\author{
Krisda H. Chaiyachati, MD, MPH, MSHP ${ }^{1,2}$, Joshua M. Liao, MD, MSc ${ }^{2,3,4}$, \\ Gary E. Weissman, MD, MSHP2,5,6, Anna U. Morgan, MD, MSC, MSHP ${ }^{2,7}$, Judy A. Shea, PhD ${ }^{1,2}$, and \\ Katrina A. Armstrong, MD, MSCE $2,8,9$ \\ 'Division of General Internal Medicine at the Perelman School of Medicine, University of Pennsylvania, Philadelphia, PA, USA; ${ }^{2}$ Leonard Davis \\ Institute of Health Economics, Philadelphia, PA, USA; ${ }^{3}$ Division of General Internal Medicine, University of Washington, Seattle, Washington, USA; ${ }^{4}$ UW \\ Medicine Value and Systems Science Lab, Seattle, Washington, USA; ${ }^{5}$ Division of Pulmonary, Allergy and Critical Care Medicine, Perelman School \\ of Medicine, University of Pennsylvania, Philadelphia, PA, USA; 'Palliative and Advanced lllness Research Center, Perelman School of Medicine, \\ University of Pennsylvania, Philadelphia, PA, USA; ${ }^{7}$ Division of General Internal Medicine, Harbor-UCLA Medical Center, California, USA; \\ ${ }^{8}$ Department of Medicine, Massachusetts General Hospital, Boston, MA, USA; ${ }^{9}$ Harvard Medical School, Boston, MA, USA.
}

J Gen Intern Med 34(3):346-8

DOI: $10.1007 / \mathrm{s} 11606-018-4702-5$

(c) Society of General Internal Medicine 2018

\section{INTRODUCTION}

Research-intensive fellowships provide early career academic generalists with research training and mentor relationships that may be important for academic career success. ${ }^{1,2}$ However, the influence of mentorship on these generalists' publication productivity, which is associated with advancement and retention in academic careers, ${ }^{3,4}$ is not known. We hypothesized that the network around mentors (i.e., how mentors are connected within a community of early career academic generalists, or "mentees") is associated with mentees' early career publications.

\section{METHODS}

Between July and August 2016, we surveyed mentees who had completed clinical training as generalists (internal medicine, pediatrics, family medicine, or combined internal medicine-pediatrics) and completed a post-residency research-intensive fellowship (general internal medicine, general pediatrics, health services, or via the Robert Wood Johnson Clinical Scholars Program) between 2002 and 2007. We identified eligible respondents and their email addresses using publicly available lists and direct contact with fellowship program directors. Using an electronic, web-based survey (REDCap), respondents were asked to report demographic information (gender, race, ethnicity) and nominate up to ten mentors they felt were particularly influential either during and/or up until five years after their research-intensive fellowship. We manually extracted the number of mentees' total and first-author peer-reviewed

Published online October 22, 2018 publications from PubMed over the same time period. Two email reminders were sent, and respondents entered a raffle for 1 of 23 Amazon gift cards (values \$10-500).

We constructed a mentor-mentee network (Fig. 1), created by linking mentors through shared mentees and mentees through shared mentors, and calculated three network characteristics: (a) mentee degrees, quantifying the total number of mentors per mentee; (b) mean mentor betweenness centrality, quantifying the importance of each mentor based on the proportion of linkages between network members for which the shortest went through that mentor (larger numbers indicate greater mentor importance); and (c) largest community membership, assigning mentees to the largest interconnected group of mentees and mentors. Using mentee-level multivariable linear regression adjusing for respondents' gender, underrepresented minority status, clinical specialty, and fellowship institution reputation (based on location at a top 25 research institution based on the 2016 US News and World Report ranking), we evaluated the association between network measures and the mentees' total number of publications and, as a secondary analysis, the number of first-author publications.

\section{RESULTS}

In total, 162 mentees representing 19 institutions completed our survey (51\% response rate) and identified 471 unique mentors for a mean (SD) of 3.8 (2.1) mentors. The majority of respondents were female $(64 \%)$, and few were underrepresented minorities $(10 \%)$. Within five years of completing fellowship, mentees had published a mean (SD) of 12.3 (11) total and 6.5 (5.4) first-author publications (Table 1). Mentees identifying mentors with higher mean betweenness centrality had more total publications $(p<0.001)$. However, mentee degrees and 


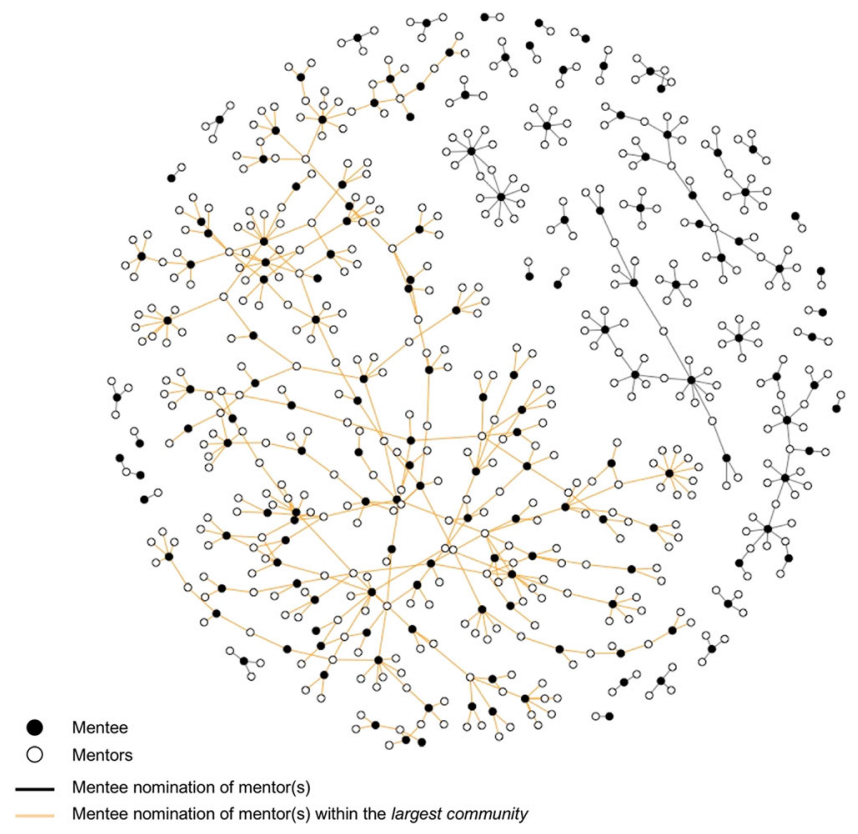

Fig. 1 Network graph of mentor-mentee community

largest community membership were not associated with the mentee's total number of publications. In secondary analyses, mean betweenness centrality and largest community membership were associated with first-author publication quantity $(p<0.001$ and $p=0.03$, respectively), but mentee degrees was not.

\section{DISCUSSION}

Understanding how mentors contribute to mentees' academic success is an important but understudied area. To our knowledge, this study is the first to identify the relationship between having mentors who are highly interconnected within the generalist community with greater peer-reviewed publication productivity, as well as the lack of relationship between other mentorship measures, such as the number of mentors and publication productivity. Our study has several limitations. First, the constructed mentor-mentee network is sensitive to the survey response rate and the accessibility of information on graduates of research-intensive fellowships. Our findings might differ with more respondents and identified mentors. Second, our analysis did not include other factors, such as mentees' professional and familial obligations (e.g., caretaking for aging parents or children), that could reduce the time and ability to build mentorship relationships or complete peer-reviewed publications. ${ }^{5,} 6$ Additional evaluations are needed to describe the multi-faceted and dynamic nature of mentor-mentee relationships, as well as the association between mentorship and publications or other forms of academic success. Future work should also validate or test additional network measures quantifying mentorship from mentees' and mentors' perspectives. Nonetheless, our study demonstrates the ability to use network approaches to address important issues regarding the relationship between mentors and success for early career academic generalists.

Table 1 Characteristics of survey respondents and adjusted models evaluating the association between the number of publications and mentorship network features

\begin{tabular}{|c|c|c|}
\hline Characteristics of survey respondents $(n=162)$ & Value & \\
\hline Female, $n(\%)$ & $104(64)$ & \\
\hline Underrepresented minority, $n(\%)$ & $16(10)$ & \\
\hline Fellowship at top 25 institution,* $n(\%)$ & $136(84)$ & \\
\hline \multicolumn{3}{|l|}{ Specialty, $n(\%)$} \\
\hline Internal medicine & $95(59)$ & \\
\hline Pediatrics & $43(27)$ & \\
\hline Family medicine & $22(14)$ & \\
\hline Medicine-pediatrics & $1(1)$ & \\
\hline Total publications, $* *$ mean $(\mathrm{sd})$ & $12.3(11)$ & \\
\hline First-author publications, ${ }^{* *}$ mean $(\mathrm{sd})$ & $6.5(5.4)$ & \\
\hline \multicolumn{3}{|l|}{ Mentor-mentee network features } \\
\hline $\begin{array}{l}\text { Mentee degrees, mean (sd) } \\
\text { Definition: total number of mentors identified }\end{array}$ & $3.8(2.1)$ & \\
\hline $\begin{array}{l}\text { Betweenness centrality of the mentor, mean }(\mathrm{sd}) \\
\text { Definition: interconnectedness of the mentors, range } 0-100\end{array}$ & $6.9(12.2)$ & \\
\hline $\begin{array}{l}\text { Largest community, } n(\%) \\
\text { Definition: belonging to the largest sub-community within the network }\end{array}$ & $98(60)$ & \\
\hline Association between total number of publications and network features & Coefficient $(95 \% \mathrm{CI})$ & $p$ value \\
\hline Mentee degrees & $0.67(-0.19$ to 1.53$)$ & 0.13 \\
\hline Mean betweenness centrality of the mentor(s) & $0.25(0.11$ to 0.39$)$ & $<0.001$ \\
\hline Largest community membership & $3.43(-0.19$ to 7.06$)$ & 0.06 \\
\hline \multicolumn{3}{|l|}{ Association between number of first-author publications and network features } \\
\hline Mentee degrees & $0.25(-0.16$ to 0.66$)$ & 0.23 \\
\hline Mean betweenness centrality of the mentor(s) & $0.12(0.06$ to 0.19$)$ & $<0.001$ \\
\hline Largest community membership & $1.97(0.25$ to 3.69$)$ & 0.03 \\
\hline
\end{tabular}

Linear regression models were used to test, separately, the association between either the total number of publications or the number of first-author publications and each of the three network variables: mentee degrees, mean betweenness centrality of mentor(s), and largest community membership. All models controlled for the gender of the mentees, the underrepresented minority status, completing fellowship at a top 25 institution based on the 2016 US News and World Report ranking of research institutions (*), and the specialty of the mentee. The total number of publications and the number of first-author publications during and within five years of completing the research-intensive fellowship (**) were collected for each mentee using PubMed. 
Corresponding Author: Krisda H. Chaiyachati, MD, MPH, MSHP; Division of General Internal Medicine at the Perelman School of Medicine University of Pennsylvania, 423 Guardian Drive, 13th Floor Blockley Hall, Philadelphia, PA 19104, USA (e-mail: kchai@upenn. edu).

\section{Compliance with Ethical Standards:}

Conflicts of Interest: The authors declare that they do not have a conflict of interest.

\section{REFERENCES}

1. DeCastro R, Sambuco D, Ubel PA, Stewart A, Jagsi R. Mentor networks in academic medicine: moving beyond a dyadic conception of mentoring for junior faculty researchers. Acad Med. 2013;88(4):488-496.
2. Steiner JF, Lanphear BP, Curtis $\mathbf{P}, \mathbf{V u}$ KO. The training and career paths of fellows in the National Research Service Award (NRSA) Program for Research in Primary Medical Care. Acad Med. 2002;77(7):712-718.

3. Carr PL, Raj A, Kaplan SE, Terrin N, Breeze JL, Freund KM. Gender differences in academic medicine: retention, rank, and leadership comparisons from the National Faculty Survey. Acad Med. 2018

4. Blazey-Martin D, Carr PL, Terrin N, et al. lower rates of promotion of generalists in academic medicine: a follow-up to the National Faculty Survey. J Gen Intern Med. 2017;32(7):747-752.

5. Girod SC, Fassiotto M, Menorca R, Etzkowitz H, Wren SM. Reasons for faculty departures from an academic medical center: a survey and comparison across faculty lines. BMC Med Educ. 2017;17(1):8.

6. The National Academies of Sciences E, and Medicine. The arc of the academic research career: issues and implications for U.S. science and engineering leadership: summary of a workshop. Washington (DC) 2014 . 